\title{
A Mysterious Phenomenon in the Field of Contemporary Immunology
}

\section{Kimihiko Okazaki*}

Okazaki Medical Clinic, Ukyoku Kyoto Japan

The concept of existence of equilibrium state among antibody molecules in the vicinity of the receptors was first proposed as early as 1965 [1]. What could be deduced from this concept is that mutual replacements of antibody molecules on the receptors are always taking place. The basis underlying the above mentioned deduction is that every antibody molecule in the vicinity of the receptors keeps repeating attaching to and detaching from receptors. On the other hand, there are facts that all of immunology-related diseases except AIDS are caused by specific antibodies. In more detail, all of allergic diseases are caused by allergen-specific antibodies while all of autoimmune diseases are caused by organ-specific antibodies. Applying the concept mentioned above to treatment of all of immunology-related diseases except AIDS, specific antibodies, that are responsible for the occurrence of the diseases, could be replaced from receptors on cell-surfaces, surface of mast cells in cases of allergic diseases and surface of cytolytic $\mathrm{T}$ lymphocytes in cases of autoimmune diseases. As a result of the above-mentioned replacements of antibodies, causes of diseases could be eliminated simply because combinations of specific antibodies with those cells, mast cells and cytolytic T lymphocytes, are the causes of the diseases. Nevertheless, the reality is that the traditional concept seems to be taken for granted to be relevant. According to the traditional concept, every antibody molecule rigidly adheres to its receptor. It seems to me that it is not very meaningful to try to explain the reason why the above mentioned application of the equilibrium state to clinical practice has not been proposed by any Immunologist, yet. This is the reason why I chose "A mysterious phenomenon in the field of contemporary immunology" as this short commentary's title.

\section{Reference}

1. Andrews $P$ (1965) The gel-filtration behavior of proteins related to their molecular weights over a wide range. Biochem J 96: 595-606.
*Corresponding author: Kimihiko Okazaki, Okazaki Medical Clinic, Ukyoku Kyoto Japan; Tel: +81-75-753-7531; E-mail: ma13081x@ma1.seikyou.ne.jp

Received July 19, 2017; Accepted July 24, 2017; Published July 29, 2017

Citation: Okazaki K (2017) A Mysterious Phenomenon in the Field of Contemporary Immunology. J AIDS Clin Res 8: 716. doi: 10.4172/2155-6113.1000716

Copyright: ( 2017 Okazaki K. This is an open-access article distributed under the terms of the Creative Commons Attribution License, which permits unrestricted use, distribution, and reproduction in any medium, provided the original author and source are credited. 